\title{
Anxiety after completion of treatment for early-stage breast cancer: a systematic review to identify candidate predictors and evaluate multivariable model development
}

\author{
Jenny Harris ${ }^{1}$ (D) $\cdot$ Victoria Cornelius $^{2} \cdot$ Emma Ream $^{3} \cdot$ Katy Cheevers $^{1} \cdot$ Jo Armes $^{1}$
}

Received: 24 November 2016 / Accepted: 31 March 2017/Published online: 12 April 2017

(C) The Author(s) 2017. This article is an open access publication

\begin{abstract}
Purpose The purpose of this review was to identify potential candidate predictors of anxiety in women with early-stage breast cancer $(\mathrm{BC})$ after adjuvant treatments and evaluate methodological development of existing multivariable models to inform the future development of a predictive risk stratification model (PRSM).

Methods Databases (MEDLINE, Web of Science, CINAHL, CENTRAL and PsycINFO) were searched from inception to November 2015. Eligible studies were prospective, recruited women with stage $0-3 \mathrm{BC}$, used a validated anxiety outcome $\geq 3$ months post-treatment completion and used multivariable prediction models. Internationally accepted quality standards were used to assess predictive risk of bias and strength of evidence.

Results Seven studies were identified: five were observational cohorts and two secondary analyses of RCTs. Variability of measurement and selective reporting precluded meta-analysis. Twenty-one candidate predictors were identified in total. Younger age and previous mental health problems were identified as risk factors in $\geq 3$ studies. Clinical variables (e.g.
\end{abstract}

Electronic supplementary material The online version of this article (doi:10.1007/s00520-017-3688-6) contains supplementary material, which is available to authorized users.

Jenny Harris

jenny.harris@kcl.ac.uk

1 Florence Nightingale Faculty of Nursing and Midwifery, King's College London, 57 Waterloo Road, London SE1 8WA, UK

2 Imperial Clinical Trials Unit (ICTU), School of Public Health, Faculty of Medicine, Imperial College London, London, UK

3 School of Health Sciences, Faculty of Health and Medical Sciences, University of Surrey, Guildford, UK treatment, tumour grade) were not identified as predictors in any studies. No studies adhered to all quality standards.

Conclusions Pre-existing vulnerability to mental health problems and younger age increased the risk of anxiety after completion of treatment for BC survivors, but there was no evidence that chemotherapy was a predictor. Multiple predictors were identified but many lacked reproducibility or were not measured across studies, and inadequate reporting did not allow full evaluation of the multivariable models. The use of quality standards in the development of PRSM within supportive cancer care would improve model quality and performance, thereby allowing professionals to better target support for patients.

Keywords Breast cancer · Anxiety $\cdot$ Predictors $\cdot$ Risk . Supportive care $\cdot$ Systematic review

\section{Introduction}

Increasing numbers of women are living with the consequences of breast cancer and its treatments [1, 2]. In many countries, cancer services are facing the challenge of meeting breast cancer survivors' (BCS) ongoing supportive care needs [3], with around a third of BCS reporting unmet psychological supportive care needs, specifically relating to anxiety, worries about their cancer diagnosis and treatment, and fears of cancer recurrence [4-6].

Anxiety is one of the most prevalent mental health symptoms experienced by BCS [7-9] and is characterised by unpleasant feelings of threat or potential harm, often accompanied by disruptive physical (e.g. muscular tension, palpitations, sweating) and cognitive (e.g. restlessness, lack of concentration) sensations $[10,11]$, which may also present alongside depressive symptoms. Although heightened anxiety is an 
understandable and often adaptive response to an initial cancer diagnosis, these symptoms may become clinically important when severity is sustained over time and worry becomes counterproductive and debilitating, affecting everyday life [12]. An estimated $20-58 \%$ of women with breast cancer (BC) experience symptoms of anxiety around diagnosis and during treatments $[13,14]$, sometimes described as 'anticipatory anxiety' and partly due to the inherent threat and uncertainly posed by a BC diagnosis and commencement of treatments [13]. Whilst for most BCS experiencing anxiety these symptoms will begin to lessen post-treatment, $18-33 \%$ will have symptoms many months and even years after treatment has ended $[12,15]$. This is of particular importance in countries, such as the UK, where there is a move away from follow-up care delivered by cancer services for BCS at low risk of cancer recurrence and late effects (physical and psychological), towards self-care and follow-up in primary care by general practitioners (GPs), despite the current paucity of evidence or tools to inform assessment of risk for psychological late effects in BCS [16].

Potential predictors of anxiety for BCS after treatment are unclear and relatively under-researched with little systematic assessment of the available evidence. Previous reviews explored efficacy of treatments for anxiety in BCS [17, 18], prevalence of anxiety across different tumour groups [11, 15] and factors associated with anxiety for women with $\mathrm{BC}$ throughout diagnosis and treatment [14, 19].

A comprehensive review suggested that younger age was associated with an increased risk of experiencing anxiety during treatment and some evidence that anxiety may be higher amongst women who had undergone more than one type of cancer treatment, and in particular chemotherapy, but found no variation for those receiving radiotherapy or by type of surgery (e.g. mastectomy vs lumpectomy) [14]. However, the focus of Lim et al.'s review was not to identify potential predictors of anxiety after treatment, and many of the studies included were of mixed quality and design (cross-sectional and longitudinal). This is important because cross-sectional studies are not designed or able to tell us what characteristics of the individual, their experience and clinical history influence future outcomes. Instead, they suggest interesting associations $[20,21]$. Further, only longitudinal studies which report multivariable analysis, where more than one independent variables' (predictors) association with a dependent variable (outcome) is analysed simultaneously in a statistical model [22], are able to identify which predictors have the strongest association with the outcome whilst adjusting for potential confounding factors [23]. Data from such studies can be used in the development of predictive risk stratification models (PRSMs), designed to predict an individual's risk of having — or developing - a specific condition or outcome based on multiple variables $[24,25]$. Being able to stratify according to risk would be an important step to ensuring that cancer and supportive services are more effectively and efficiently tailored to identify the future risk of anxiety, alongside any current need, in individual BCS and thereby has the potential to improve the support they receive [16].

The development of PRSM requires identification of potential candidate predictor variables and should be informed by risk prediction systematic review [26], focused on identifying studies that use longitudinal designs and multivariable analysis to distinguish the variables most strongly associated with an outcome [24, 27-29]. Therefore, the aim of this review is to identify candidate predictors of post-treatment anxiety for women with early-stage (0-3) breast cancer after completion of post-surgical/adjuvant treatments. Candidate predictors were assessed and described using recently developed, internationally accepted standards for assessing the quality and strength of evidence in risk prediction research [30, 31]. Findings from this review will inform the development of a PRSM of anxiety for use in clinical practice.

\section{Methods}

\section{Literature search and eligibility criteria}

Electronic searches were undertaken in MEDLINE, Web of Science, CINAHL, CENTRAL and PsycINFO (inception to November 2015) using a search strategy informed by previously published strategies [32-34] and included terms for breast cancer, anxiety and predictive studies (supplementary material 1). Extensive cross-referencing of selected studies was undertaken as well as searches of reference lists of previously published literature reviews [11, 14, 15, 17-19]. The search strategies from each electronic database were saved and results downloaded to EndNote X7.5 for electronic bibliographic management. The protocol was submitted to the PROSPERO register [35].

This review included published journal articles of prospective or retrospective longitudinal studies examining anxiety after treatment in women with early-stage (0-3) breast cancer with a baseline measure of predictive factors and at least 3 months' follow-up after completion of treatment. Eligible designs included case-control, survey or cohort studies, analysis of registry data and secondary analysis of prospective studies (e.g. RCT of cancer treatments). Eligible studies included samples of adult ( $\geq 18$ years) women diagnosed with stage $0-3$ breast cancer who underwent surgical and (neo)adjuvant treatments. Treatment was defined as including surgical, radiological therapies and chemotherapy (adjuvant and neo-adjuvant). Women receiving hormone or biological therapies were included in the population of interest but not included in the definition of treatment, as such treatments can continue for many years after primary and adjuvant treatments 
have ceased and would thereby exclude too many participants and limit the generalisability and usefulness of the results.

Eligible studies needed to include an outcome of anxiety symptoms or disorders, measured at least 3 months after active treatment for non-metastatic $\mathrm{BC}$ using a validated screening tool or diagnostic tool (i.e. consistent with DSM-IV or ICD-10 criteria), including, but not limited to, measures such as the State-Trait Anxiety Inventory (STAI) [36], Beck Anxiety Inventory (BAI) [37] and Hospital Anxiety and Depression Scale-Anxiety (HADS-A) [38], or diagnostic interviews such as the Structured Clinical Interview (SCID-II) [39]. To ensure all relevant data were included, if a study reported combined outcomes, for example, coefficient estimates for mixed anxiety and depression symptoms, they were included in the review.

The minimum follow-up period for inclusion was at least 3 months after all baseline (predictor) data collection and treatment (as defined above) was complete. This was chosen because in the EU, the USA and elsewhere, this usually coincides with the first clinical follow-up appointment when written care plans, including continued follow-up in primary, secondary or shared care, are agreed. Additionally, at this point, referrals for specialist care and support services can also be made [40-42]. Full eligibility criteria are available from the protocol.

In two instances, multiple publications from the same primary study were found [43, 44]. In line with previous risk prediction reviews [45], in such cases, the authors compared the competing studies to identify the most comprehensive/ relevant adjustment and outcome measures, and duration of follow-up, with the publication meeting the most criteria being included in the review.

\section{Data extraction}

Two reviewers (JH, KC) independently extracted data. A standardised data extraction form designed specifically for systematic reviews of prediction models, the CHecklist for critical Appraisal and data extraction for systematic Reviews of prediction Modelling Studies (CHARMS) [28], was adapted for this review. Data extracted included publication details, sample size, prevalence of outcome (where appropriate), candidate predictors and outcome measurement, including length of follow-up. Where available, coefficients, standard errors, $p$ values, odds or hazard ratios, and confidence intervals were extracted for the final multivariable model and for each time point. It was noted if potential candidate predictor data or covariates included in the final model were not reported.

Candidate predictors were grouped according to three domains: (1) socio-demographic, (2) biomedical and clinical, and (3) psychosocial and lifestyle behaviours. A similar approach has been used in other reviews [46]. Whilst it is acknowledged there is inevitably some overlap between these three domains, this approach was considered helpful to aid interpretation of the findings by helping to determine the quality and depth of evidence in different areas in order to inform clinical practice, future research and development of conceptual models of anxiety amongst BCS. For completeness, data on time-dependent covariates, that is, a variable that is repeatedly measured during the study, were also extracted.

\section{Assessing risk of bias and strength of evidence}

The two reviewers $(\mathrm{JH}, \mathrm{KC})$ assessed the methodological quality of all included studies with the validated Quality in Prognostic Studies (QUIPS) tool developed by the Cochrane Collaboration Prognostic Methods Group [30]. QUIPS evaluates the risk of bias in six domains: study participants, study attrition, prognostic factor measurement, outcome measurement, study confounding, and statistical analysis and reporting. Usefully, QUIPS allows for both statistical and narrative sensitivity analysis to be undertaken to assess the impact of study quality on the study findings. In addition, a modified version of the Grading of Recommendations Assessment, Development and Evaluation (GRADE) framework [47], recommended specifically for risk prediction systematic reviews [31], was used to assess the strength of evidence for identified predictors (see supplementary material 2). This framework identifies six domains that can reduce the quality of evidence (phase of investigation, study limitations (incorporating QUIPS risk of bias), inconsistency, indirectness, imprecision, publication bias) and two that can increase the rating (moderate or large effect size, dose (exposure-response) gradient). Disagreements were resolved through discussion with third reviewers (JA or VC).

\section{Data analysis}

Heterogeneity in study methodology precluded meta-analysis. We describe the individual candidate predictors and outcome measures from the identified studies. Almost all studies reported only adjusted estimates; therefore, these are reported where available. We also describe the statistical modelling methods used in order to fully assess the quality of analysis and strength of evidence.

\section{Results}

\section{Identification of studies}

In total, 2465 records were initially identified (2456 through databases and nine from cross-referencing) (Fig. 1). After removing duplicates, 1373 abstracts were initially screened, and of these, 1180 studies were excluded because the design, 
Fig. 1 Identification of studies for the systematic review

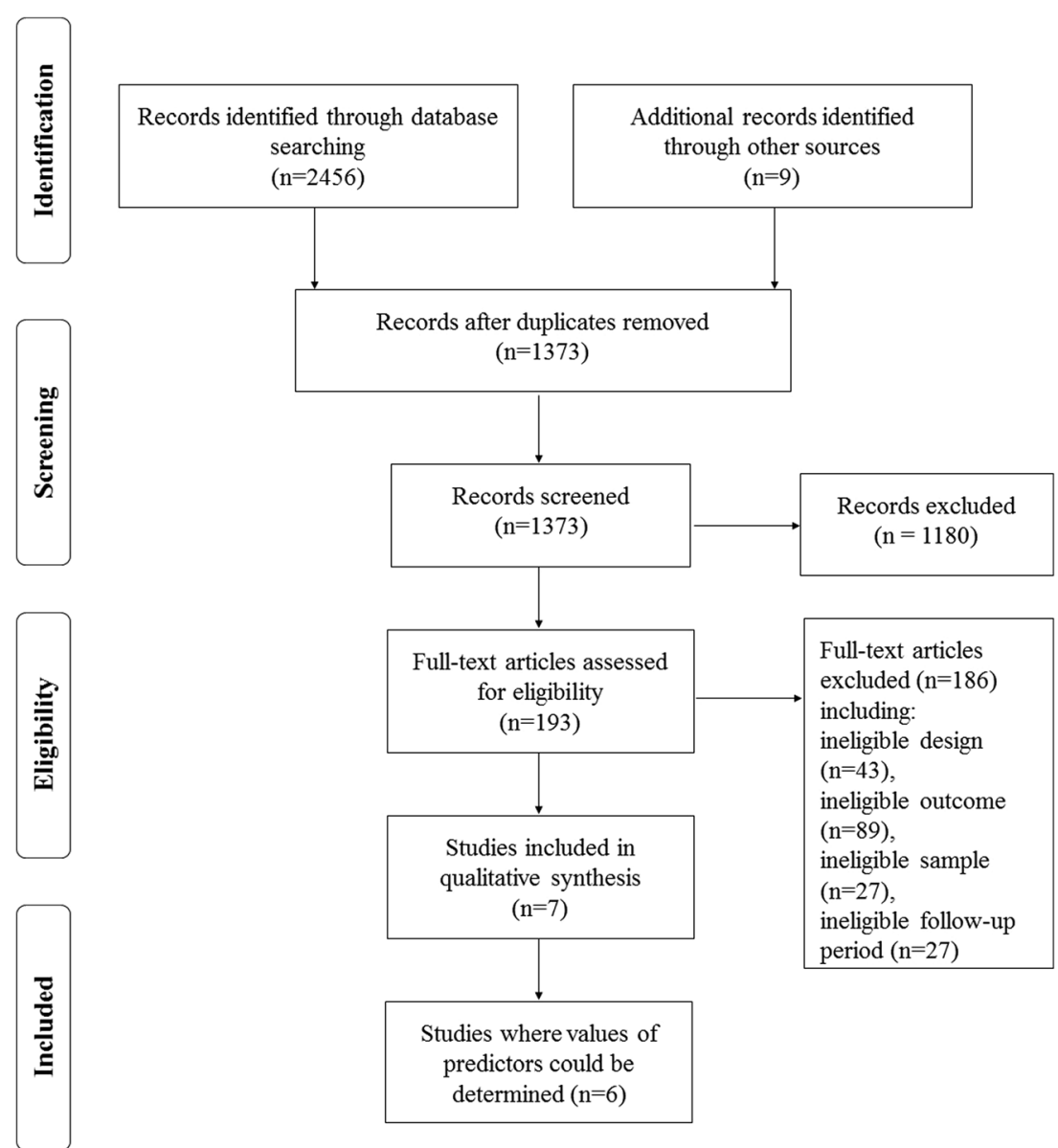

sample and outcome were ineligible, resulting in 193 full-text articles being obtained and assessed further for eligibility. Overall, 43 were ineligible due to design or focus, 27 due to samples, 89 due to outcome measures and 27 due to followup. A final total of seven studies were included and are described.

\section{Study characteristics}

The total sample size was 3238, ranging from 56 to 1801 participants per study (Table 1 ). The mean age ranged from 48.4 to 56.5 years; however, one study did not provide detailed age data [48] and two specified upper and/or lower age limits as part of their inclusion criteria $[49,50]$. Four samples were from the UK $[48,49,51,52]$, and one each was from Sweden [50], Portugal [53] and Hong Kong [54]. Five studies used observational cohort designs [48-51, 53, 54], and two undertook secondary analysis of RCT data [51, 52].

Studies used face-to-face interviews [48, 49, 52], selfcompleted surveys $[50,51,53]$ or a combination of face-toface and telephone interviews [54]. The duration and timing of the follow-up period after treatment completion ranged from
6 months to 6 years (Table 1), with only two studies including repeated measurements of the outcome $[49,51]$.

\section{Outcome measures}

Five studies used the outcome of anxiety [48, 50, 51, 53, 54], whereas two presented results for mixed anxiety and depression [49, 52] (Table 1).

Four studies each used the HADS-A [38], including translated versions, where appropriate. Two of these studies used the HADS-A as a continuous outcome measure [53, 54] and so did not report prevalence (Table 1). Hopwood et al. [51] reported a borderline anxiety rate of $16 \%$ and a probable case rate of $13 \%$ at 5 years, and Saboonchi et al. [50] reported $25 \%$ possible or probable anxiety cases at 12 months after surgery.

Three studies used different clinical outcome measures (the Structured Clinical Interview [49], Spitzer's Research Diagnostic Criteria [52] and the Schedule for Affective Disorders and Schizophrenia [48]). Burgess et al. reported a cumulative prevalence of $48 \%$ full or borderline cases of anxiety and/or depression from diagnosis up until 12 months, with point prevalence reducing to $25 \%$ at 2 years, $23 \%$ at 3 years, $22 \%$ at 4 years and $15 \%$ at 5 years [49]. Dean found a point 


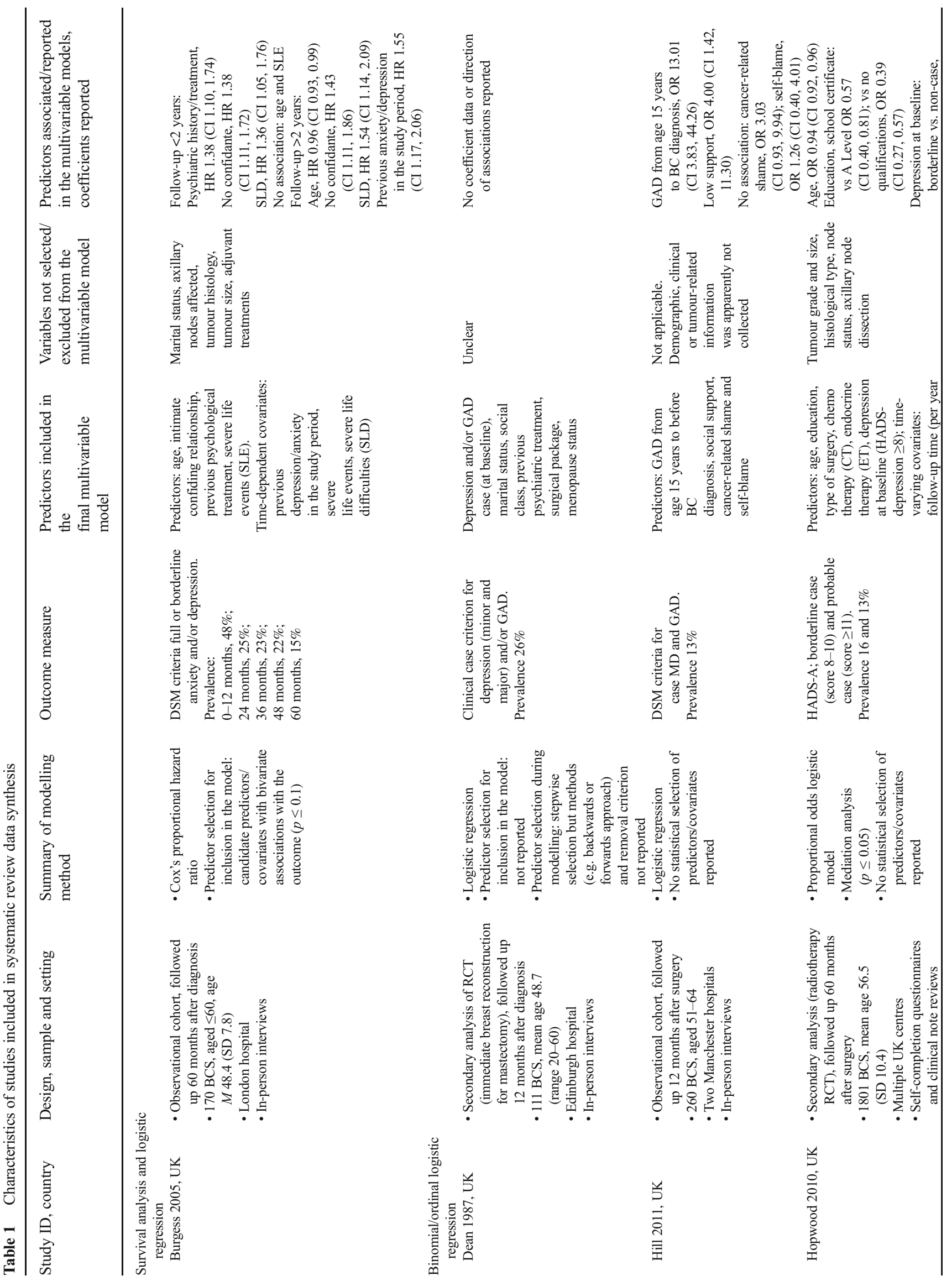




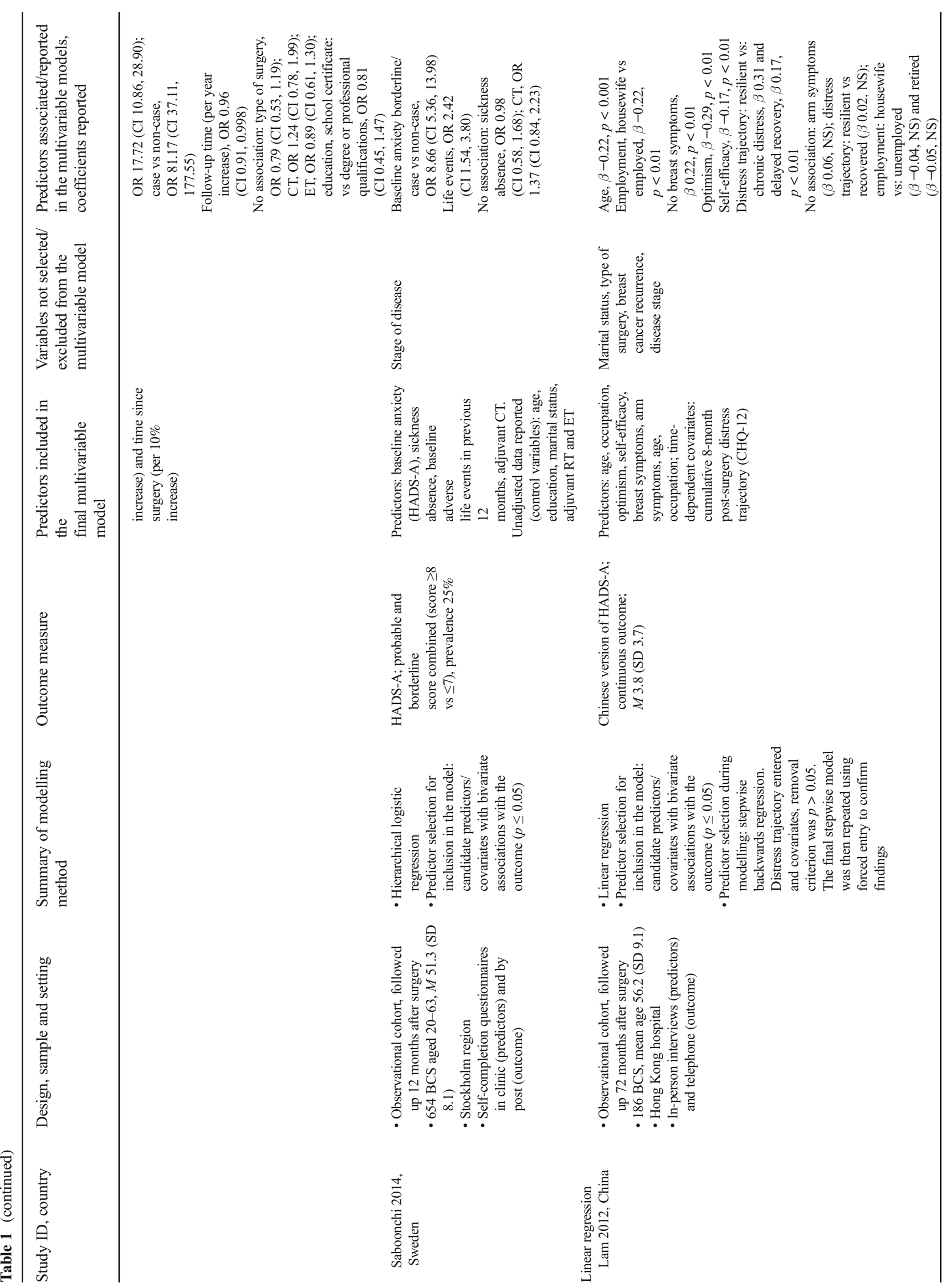


prevalence of depression and/or anxiety of $26 \%$ at 12 months [52], and Hill et al. reported $13 \%$ with a diagnosis of generalised anxiety disorder during the first year after diagnosis [48].

\section{Statistical modelling approaches}

Three types of prediction modelling were used. Burgess et al. [49] used time-to-event modelling with stressful life events as a time-dependent variable and depression/anxiety as a binary outcome $(\mathrm{Y} / \mathrm{N})$ for medium-term outcome ( 4 months to 2 years after diagnosis); all other predictors were measured at diagnosis. Logistic regression was used to predict longer term outcome (from 2 to 5 years after diagnosis). All potential predictors that indicated significance at the $p \leq 0.1$ level were included in the final model.

Four other studies used only logistic models. Saboonchi et al. [50] used hierarchical logistic regression (possible or probable anxiety, $\mathrm{Y} / \mathrm{N}$ ); again, the multivariable model only included predictors that demonstrated bivariate associations $(p \leq 0.05)$. Hopwood et al. [51] used proportional odds logistic regression (for ordinal data; normal, borderline or case anxiety) and did not report any specific selection criteria for inclusion in the model or during modelling. Hill et al. [48] used Baron and Kenny's [55] mediation approach whereby selection for inclusion and during modelling is based on hypothesis and bivariate associations $(p \leq 0.05)$ (Table 1$)$.

Two studies used linear multiple regression, to model the HADS-A as a continuous outcome measure at a single specified time point and predictors measured at completion of treatment, although selection methods differed. Lam et al. [54] used the stepwise, backwards method for predictor inclusion during multivariable modelling (confirmed in personal correspondence, removal criterion was $p>0.05$ ), whereas Moreira and Canavarro [53] used a hierarchical approach and only predictors significantly correlated with anxiety $(p \leq 0.05)$ were included. Dean [52] reports that predictors with a bivariate association were included in a stepwise logistic regression (mixed anxiety and depression), but specific details for this approach are not reported.

Overall, three studies reported all coefficients in the final multivariable model (i.e. regardless of significance) $[48,49$, 51], three studies only reported coefficient data for predictors found to be significant in the final model $[50,53,54]$ and one study did not report any coefficient data [52] (Table 1).

\section{Risk of bias within and across studies}

QUIPS [30] appraisal suggested the studies by Burgess et al. [49] and Hopwood et al. [51] had the lowest risk of bias (Table 2). Three studies were assessed to have high risk of bias in at least one domain $[48,52,53]$. Overall, the studies had low risk of bias in the domain of outcome measurement, meaning that they performed well as they used robust and 
Table 2 Quality in Prognostic Studies (QUIPS) risk of bias assessment for included studies

\begin{tabular}{|c|c|c|c|c|c|c|}
\hline \multirow[t]{2}{*}{ Study source } & \multicolumn{6}{|l|}{ Bias domain } \\
\hline & $\begin{array}{l}\text { Study } \\
\text { participation }\end{array}$ & $\begin{array}{l}\text { Study } \\
\text { attrition }\end{array}$ & $\begin{array}{l}\text { Prognostic } \\
\text { factor } \\
\text { measurement }\end{array}$ & $\begin{array}{l}\text { Outcome } \\
\text { measurement }\end{array}$ & $\begin{array}{l}\text { Study } \\
\text { confounding }\end{array}$ & $\begin{array}{l}\text { Statistical } \\
\text { analysis \& } \\
\text { reporting }\end{array}$ \\
\hline $\begin{array}{l}\text { Burgess et al, } \\
2005\end{array}$ & & & & & & \\
\hline $\begin{array}{l}\text { Hopwood et al, } \\
2010\end{array}$ & & & & & & \\
\hline $\begin{array}{l}\text { Saboonchi et } \\
\text { al, } 2014\end{array}$ & & & & & & \\
\hline Lam et al, 2012 & & & & & & \\
\hline Hill et al, 2011 & & & & & & \\
\hline $\begin{array}{l}\text { Moreira et al, } \\
2010\end{array}$ & & & & & & \\
\hline Dean, 1987 & & & & & & \\
\hline
\end{tabular}

validated tools which were administered in a consistent way. Study participation presented low to moderate risk of bias in all studies. Ratings of bias for study attrition, study confounding, statistical analysis and reporting, and prognostic factor measurement were more mixed.

\section{Candidate predictors}

Twenty-eight individual candidate predictors were evaluated by the studies, and, overall, 21 were included in a final multivariable model by at least one study. The remaining seven were subsequently excluded from final multivariable models due to non-significant bivariate associations, suggesting an initial exploratory analysis indicated they were indirect covariates or confounders (Table 1, supplementary material 3 ). In addition, five time-dependent covariates were identified across three studies [49, 51, 54]. Assessment of the strength of evidence for each candidate predictor is presented according to GRADE [31], discussed in the following sections (Table 3, supplementary material 3).

\section{Socio-demographic characteristics}

Five socio-demographic candidate predictors were evaluated: age, marital status, education level, occupation and sickness absence. Evidence was graded as moderate for age and low to very low for the other socio-demographic variables.

Three studies found older age reduced the probability of posttreatment anxiety $[49,51,54]$. A single study found no bivariate association [50] and so did not include age in the final

Table 3 Strength of evidence for candidate predictors identified across all included studies

\footnotetext{
High - Increased risk: previous mental health problems or treatment; baseline anxiety/depression

Moderate • No association: chemotherapy

- Inconsistent evidence: age (younger age increases risk or no association)

Low Increased risk: non-cancer life difficulties, no intimate confidante

- Reduced risk: higher self-efficacy, higher optimism

- No association: surgery, radiotherapy and hormone therapies, cancer diagnostic indicators, marital status, appearance satisfaction, cancer-related shame, sickness absence

- Inconsistent evidence: severe/adverse life events (increased risk or no association)

Very low • Reduced risk: 'housewife', no breast symptoms

- No association: self-consciousness of appearance, arm symptoms

- Inconsistent evidence: education (increased risk if less educated or no association)
} 
multivariable model, whilst for another it was not reported [53]. Four studies assessed bivariate associations between marital status and anxiety outcomes but did not include this in final multivariable models [49, 50, 53, 54]. One study reported BCS described as 'housewives' had lower levels of anxiety (compared to those who were employed/unemployed/retired) [54]. Saboonchi et al. [50] considered sickness absence from work as a candidate predictor but found no association with 12-month anxiety. Years in education or level of academic qualification were considered by three studies. Lam et al. [54] found that those with no qualifications had an increased risk of anxiety; however, the two other studies suggested no such association [51, 53].

None of the included studies considered other potentially important socio-demographic candidate predictors including socioeconomic status, income or measures of deprivation, ethnicity, religiosity, caring responsibilities or other social determinants.

\section{Biomedical and clinical characteristics}

Overall, 11 cancer-related clinical characteristics were explored and considered as potential candidate predictors. Several studies assessed treatment characteristics; however, the strength of evidence was graded low, with the exception of chemotherapy graded as moderate. Candidate treatment predictors included type of surgery (e.g. mastectomy vs breast conservation) $[51,53,54]$, axillary surgery [51, 53], endocrine therapy and chemotherapy [49-51, 53] and radiotherapy [50, 53]. None were found to predict anxiety outcome, and most studies did not include these in their multivariable analyses.

A single study considered the absence of post-surgery arm and breast symptoms, with only having no breast symptoms reducing women's risk of anxiety at 5-year follow-up [54]. No studies considered the impact of comorbidities, disability, experience of neutropenic events during systemic therapies or the experience of other side effects during treatment, as possible candidate predictors.

Diagnostic indicators, including axillary node status [51, 53], pathological tumour size [49, 51, 54], tumour grade [49, 51] and histological type [49, 53, 56], were assessed using bivariate analysis but not included in any multivariable models due to $p$ values below study thresholds. The strength of evidence was graded low.

\section{Psychosocial and behavioural lifestyle characteristics}

Patients with a previous history of mental health problems and/or psychiatric treatment were more likely to have anxiety in two studies [48, 49]. Likewise, anxiety or depression at baseline was associated with increased risk at follow-up in three studies [50, 51, 53]. Similarly, anxiety/depression as time-dependent covariates during the study period (i.e. not just at baseline but cumulatively measured up to outcome) indicated increased risk of anxiety at 5-year follow-up in one study
[54]. Due to the consistency and moderate-to-large effect sizes from multiple studies, the strength of evidence was graded moderate to high.

Several studies found no association between anxiety and psychosocial factors, including appearance satisfaction [53], cancer-related shame [48], self-blame [48] and selfconsciousness about appearance [53]. Specific predictors found to have an association included lack of an intimate confidant [49, 50], experiencing severe life difficulties [49] and low levels of social support [48], all found to increase the risk, whereas higher optimism and self-efficacy [54] reduced the risk of anxiety. However, because these characteristics were assessed in single studies, the evidence was graded very low to low. Experiencing a severe adverse life event (either prior to or soon after cancer diagnosis) was found to increase the odds of anxiety in one study [50] but showed no association in another [49], although these studies used heterogeneous measures (detailed interview assessment vs. one item, self-completion) and the evidence was graded as low. No evidence was found of eligible studies that considered healthrelated behaviours or other lifestyle-related factors (e.g. smoking, diet or physical activity).

\section{Time-dependent covariates}

With the exception of anxiety/depression during the study period, as highlighted earlier, evidence about the remaining time-dependent covariates was graded low. One study found that study follow-up time was associated with anxiety, whereby longer follow-up time reduced the risk of anxiety [51]. Lam et al. [54] found no bivariate association between recurrence of breast cancer and anxiety at 5 years. However, they report a weak dose-response association with post-surgery distress trajectories; those who were more psychologically resilient after surgery had lower levels of anxiety by 5 years. No studies considered the role of ongoing side effects or physical symptoms from surgery, radiotherapy or systematic therapies as possible time-dependent covariates.

\section{Discussion}

This is the first review to systematically synthesise available longitudinal evidence from seven studies regarding possible candidate predictors of anxiety amongst BCS after treatment has ended and they have entered follow-up care. It is notable that the evidence for many candidate predictors is weak, as revealed by this systematic review using gold-standard tools $[30,31]$ to evaluate the quality and robustness of prognostic evidence.

A growing population of BCS worldwide will have longterm symptoms of anxiety, and healthcare providers currently lack ways to identify those at greatest risk; thus, new ways to help distinguish those at greatest risk are required. 
Understanding the predictors of anxiety in BCS may be useful to inform and enhance follow-up care through heightened clinical awareness [24] or the formal use of PRSM alongside ongoing needs assessments [16].

Results indicate the risk of anxiety is greatest for those with pre-existing poor mental health and those with heighted anxiety around the time of diagnosis/treatment. Although the measures used varied, previous/baseline mental health predictors showed the strongest association with anxiety across five studies [48-51, 53]. Likewise, previous research suggests trait anxiety has a strong association with anxiety symptoms [14], but this was not assessed in any of the included studies and warrants further investigation.

In line with a previous review focused on anxiety amongst women with BC undergoing treatments [14], there was moderately strong evidence that younger BCS had an increased risk of anxiety [49, 51, 54]. However, this association was not consistently demonstrated across all studies [50,53], in line with the findings from a review of predictors of quality of life in colorectal survivors [46].

In contrast to Lim et al. [14], the review found no evidence that chemotherapy is a predictor of anxiety in BCS. This lack of association may be a reflection of the limited sample size and number of studies assessing this longitudinally, but an alternative hypothesis is that whilst chemotherapy heightens anxiety in the period initially prior to and during treatment, this is not sustained long-term. Alternatively, this lack of association could reflect the failure of previous research to include more sensitive candidate predictors such as measures of treatment side effects. Further longitudinal studies are needed to explore these issues and will be useful to health professionals seeking to reassure patients about the long-term impact of treatments.

The studies included in this review incorporated both clinical assessments of anxiety and the HADS [38]. Several studies aggregated both possible and probable (clinically significant) anxiety in their analysis [49-51], but interestingly similar important predictors emerged regardless of specific outcome measures used or whether they were treated as continuous, binomial or ordinal.

The results from this review shed light on a number of other gaps in our current knowledge of predictors of anxiety in BCS. Classifying types of predictors into different domains revealed that many potentially important social determinants found to be important predictors of anxiety in other chronic health conditions or in other cancer samples are yet to be examined longitudinally in BCS. These include prognostic factors such as income, deprivation and debt, caring responsibilities, social support, ethnicity and fertility [12]. However, a further publication [43] using the same sample as that of Saboonchi et al. [50] suggests that having children and financial difficulties are independently associated with sustained anxiety and poor adjustment during treatment and through to survivorship, and these warrant further investigation as candidate predictors.
For biomedical and clinical predictors, much of the focus to date has been on diagnostic markers and treatment indices, none of which have shown an association with anxiety outcomes. Only one study included any measurement of the side effects of treatment and this was limited to breast surgery symptoms [54], and no longitudinal multivariable evidence was available for other areas of physical functioning, comorbidities or disability.

An interesting observation from the psychosocial and lifestyles domain was the limited overlap in the constructs measured by individual studies and that few predictive associations were found. Consequently, where there was an association, such as optimism [54], the evidence was rated as weak as it came from only one study source. Future research should examine psychosocial constructs shown to have an association in this review to see if such findings can be replicated as they may offer important targets for future interventions. Similarly, no evidence was found on health and lifestyle behaviours such as smoking, exercise and alcohol consumption, which have been associated with anxiety and quality of life in other chronic health conditions and some cancer populations [57-60].

Although the statistical methods used in predictive risk research are not new, it is only relatively recently that researchers have begun to explore and understand the importance of adopting a rigorous methodological approach in developing prediction models and the need for different approaches to the development of causation models [20, 24, 61]. In developing PRSM, it is now recognised that candidate predictors may be important even if they are not considered causal and so may be included if considered clinically important, even when they do not reach statistical significance in bivariate analysis [26, 29]. In line with common practice at the time, most studies reviewed here, excepting Hopwood et al. [51], did not include potential candidate predictors in the model if they did not reach a defined level of bivariate statistical significance. Although all of the studies cited previous research as informing their study, there was little evidence of including candidate predictors from previous models, reflecting the exploratory nature of much of the research to date. Consequently, many potential candidate predictors have only been tested in a single study, making comparisons between studies and their statistical models challenging. No studies reported the development of a PRSM for use in clinical practice or external model validation either within the study or in a subsequent study.

The strength of our findings is enhanced by adherence to systematic review principles including independent data sources, systematic identification and retrieval, cross-referencing and a broad approach to literature searching informed by recommendations. However, several limitations should be noted. Only the principal author $(\mathrm{JH})$ undertook the initial screening, although the second reviewer $(\mathrm{KC})$ assessed all the abstracts retrieved. As with all systematic reviews, we cannot be certain that we did not exclude or miss some important studies; however, we tried to minimise this with assessment of full-text articles for further studies. 
Unlike previous reviews identifying predictors of psychological outcomes in cancer patients $[14,46]$, a key strength of this review is that it focused on longitudinal data, albeit with varying length of follow-up (6-72 months after completion of treatment); nevertheless, this did reduce the number of available data sources. However, this was necessary to get an accurate picture of the current strength of evidence in this area, where associations from cross-sectional studies are often inaccurately described as predictors, even in article titles. This is important because cross-sectional research, whilst identifying useful preliminary associations, may be influenced by a form of Neyman's bias, whereby an apparent association is spurious and where it is not possible to determine the direction of influence [21]. Nevertheless, it should be acknowledged that studies with an outcome of combined anxiety and depression were eligible, as we felt it was important to include such studies as many BCS will experience combined anxiety and depression. Subsequent narrative sensitivity analysis suggested that the exclusion of these studies would not have changed the overall finding of the review. A further limitation of the evidence generally is the predominance of White-European participants, with only one study from Asia and none from the Americas, Middle East or African countries [12]. It is likely that predictors of anxiety in BCS vary both within and across country by important social, cultural and healthcare contextual determinants, but as yet such evidence is unavailable. Additionally, two studies included age limitations in their recruitment criteria [49,50], and younger BCS are likely to have been overrepresented. It will be important to unpick how such risk profiles vary in future research as well as to explore whether candidate predictors vary by location of the primary cancer and if they vary for other mental health outcomes in BCS (e.g. depression, psychological distress and fear of recurrence)

The findings of this review will be used to inform the development of a PRSM to individuals at risk of developing, or continuing to experience, symptoms of anxiety after completion of treatment for breast cancer. The next steps will involve statistical development and validation of the model. A key consideration for the selection of predictors for inclusion in a model will be the extent to which they can be readily collected as part of routine clinical practice - importantly, both pre-existing vulnerability to mental health problems and age should meet this requirement. Future research will be needed to determine how best to implement and evaluate whether healthcare professionals and patients use PRSM in oncology practice, to ensure more appropriate, informed assessment of psychosocial need, thus promoting person-centred care and enhanced service provision [24, 29].

\section{Conclusions}

We found evidence that pre-existing vulnerability to mental health problems and younger age increased the risk of anxiety after treatment for BCS, but that being treated with chemotherapy probably does not. We have identified many gaps and inadequacies in the existing research evidence regarding potential predictors of anxiety in BCS after treatment. This review highlights the lack of validated and well-tested risk prediction models for use in clinical practice. The use of quality standards in the development of PRSM within supportive cancer care may improve model quality and performance [62], thereby allowing professionals to better target support for patients. These results will be used to develop and validate a PRSM with the aim to develop a tool for healthcare professionals to identify and prioritise interventions for those at greatest risk of long-term anxiety.

\section{Compliance with ethical standards}

Funding This research received no specific grant from any funding agency. Open access for this article was funded by King's College London.

Conflict of interest The authors declare that they have no conflict of interest.

Ethical approval This article does not contain any studies with human participants or animals performed by any of the authors.

Open Access This article is distributed under the terms of the Creative Commons Attribution-NonCommercial 4.0 International License (http:// creativecommons.org/licenses/by-nc/4.0/), which permits any noncommercial use, distribution, and reproduction in any medium, provided you give appropriate credit to the original author(s) and the source, provide a link to the Creative Commons license, and indicate if changes were made.

\section{References}

1. Santin O, Mills M, Treanor C, Donnelly M (2012) A comparative analysis of the health and well-being of cancer survivors to the general population. Supp Care Cancer 20(10):2545-2552

2. National Cancer Survivorship Initiative (2013) Living with and beyond cancer: taking action to improve outcomes. London: NHS Improvement, Department of Health, Macmillan Cancer Support

3. Richards M, Corner J, Maher J (2011) The National Cancer Survivorship Initiative: new and emerging evidence on the ongoing needs of cancer survivors. Br J Cancer 105:S1-S4

4. Armes J, Crowe M, Colbourne L, Morgan H, Murrells T, Oakley C et al (2009) Patients' supportive care needs beyond the end of cancer treatment: a prospective, longitudinal survey. J Clin Oncol 27(36):6172-6179

5. Sarkar S, Sautier L, Schilling G, Bokemeyer C, Koch U, Mehnert A (2015) Anxiety and fear of cancer recurrence and its association with supportive care needs and health-care service utilization in cancer patients. J Cancer Surviv 9(4):567-575

6. Fiszer C, Dolbeault S, Sultan S, Brédart A (2014) Prevalence, intensity, and predictors of the supportive care needs of women diagnosed with breast cancer: a systematic review. Psycho-Oncology 23(4):361-374

7. Mehnert A, Brähler E, Faller H, Härter M, Keller M, Schulz H et al (2014) Four-week prevalence of mental disorders in patients with cancer across major tumor entities. J Clin Oncol 32(31):3540-3546 
8. Linden W, Vodermaier A, MacKenzie R, Greig D (2012) Anxiety and depression after cancer diagnosis: prevalence rates by cancer type, gender, and age. J Affect Disord 141(2-3):343-351

9. Brintzenhofe-Szoc KM, Levin TT, Li Y, Kissane DW, Zabora JR (2009) Mixed anxiety/depression symptoms in a large cancer cohort: prevalence by cancer type. Psychosomatics 50(4):383-391

10. Stark DPH, House A (2000) Anxiety in cancer patients. Brit J Cancer 83(10):1261

11. Mitchell AJ, Chan M, Bhatti H, Halton M, Grassi L, Johansen C et al (2011) Prevalence of depression, anxiety, and adjustment disorder in oncological, haematological, and palliative-care settings: a meta-analysis of 94 interview-based studies. Lancet Oncol 12(2): 160-174

12. Remes O, Brayne C, Linde R, Lafortune L (2016) A systematic review of reviews on the prevalence of anxiety disorders in adult populations. Brain Behav 6(7):e00497. doi:10.1002/brb3.497

13. Jacobsen PB, Bovbjerg DH, Redd WH (1993) Anticipatory anxiety in women receiving chemotherapy for breast cancer. Health Psychol 12(6):469-475

14. Lim CC, Devi MK, Ang E (2011) Anxiety in women with breast cancer undergoing treatment: a systematic review. Int J Evid Based Healthc 9(3):215-235

15. Maass SW, Roorda C, Berendsen AJ, Verhaak PF, de Bock GH (2015) The prevalence of long-term symptoms of depression and anxiety after breast cancer treatment: a systematic review. Maturitas 82(1):100-108

16. Watson E, Rose PW, Neal RD, Hulbert-Williams N, Donnelly PK, Hubbard G et al (2012) Personalised cancer follow-up: risk stratification, needs assessment or both? Brit J Cancer 106(1):1-5

17. Osborn RL, Demoncada AC, Feuerstein M (2006) Psychosocial interventions for depression, anxiety, and quality of life in cancer survivors: meta-analyses. Int J Psychiatry Med 36(1):13-34

18. Sheard T, Maguire P (1999) The effect of psychological interventions on anxiety and depression in cancer patients: results of two meta-analyses. Brit JCancer 80(11):1770

19. Montgomery M, McCrone SH (2010) Psychological distress associated with the diagnostic phase for suspected breast cancer: systematic review. J Adv Nurs 66(11):2372-2390

20. Hemingway H, Croft P, Perel P, Hayden JA, Abrams K, Timmis A et al (2013) Prognosis research strategy (PROGRESS) 1: a framework for researching clinical outcomes. BMJ 346:e5595

21. Grimes DA, Schulz KF (2002) Bias and causal associations in observational research. Lancet 359(9302):248-252

22. Hidalgo B, Goodman M (2013) Multivariate or multivariable regression? Am J Public Health 103(1):39-40

23. Tolles J, Meurer WJ (2016) Logistic regression: relating patient characteristics to outcomes. JAMA 316(5):533-534

24. Moons KG, Royston P, Vergouwe Y, Grobbee DE, Altman DG (2009) Prognosis and prognostic research: what, why, and how? BMJ 338:b375. doi:10.1136/bmj.b375

25. Hutchings HA, Evans BA, Fitzsimmons D, Harrison J, Heaven M, Huxley P et al (2013) Predictive risk stratification model: a progressive cluster-randomised trial in chronic conditions management (PRISMATIC) research protocol. Trials 14(1):301

26. Pavlou M, Ambler G, Seaman SR, Guttmann O, Elliott P, King M et al (2015) How to develop a more accurate risk prediction model when there are few events. BMJ 351:h3868

27. Hayden JA, Chou R, Hogg-Johnson S, Bombardier C (2009) Systematic reviews of low back pain prognosis had variable methods and results - guidance for future prognosis reviews. J Clin Epidemiol 62(8):781-96.e1. doi:10.1016/j.jclinepi.2008.09.004

28. Moons KG, de Groot JA, Bouwmeester W, Vergouwe Y, Mallett S, Altman DG et al (2014) Critical appraisal and data extraction for systematic reviews of prediction modelling studies: the CHARMS checklist. PLoS Med 11(10):e1001744
29. Royston P, Moons KGM, Altman DG, Vergouwe Y (2009) Prognosis and prognostic research: developing a prognostic model. BMJ 338. doi:10.1136/bmj.b604

30. Hayden JA, van der Windt DA, Cartwright JL, Côté P, Bombardier C (2013) Assessing bias in studies of prognostic factors. Ann Intern Med 158(4):280-286

31. Iorio A, Spencer FA, Falavigna M, Alba C, Lang E, Burnand B et al (2015) Use of GRADE for assessment of evidence about prognosis: rating confidence in estimates of event rates in broad categories of patients. BMJ 350. doi:10.1136/bmj.h870

32. Altman DG (2001) Systematic reviews of evaluations of prognostic variables. BMJ 323(7306):224-228

33. Cochrane Breast Cancer Review Group (2015) Electronic searches in search strategies. http://onlinelibrary.wiley.com/o/cochrane/ clabout/articles/BREASTCA/frame.html. Accessed 11 Nov 2016

34. Cochrane Common Mental Disorder Review Group (2015) Search strategies for the identification of studies. http://cmd.cochrane.org/ search-strategies-identification-studies. Accessed 11 Nov 2016

35. Harris J, Cornelius V, Ream E, Cheevers K, Armes J (2016) Predicting symptoms of anxiety after breast cancer treatment: a protocol for a meta-analysis and systematic review. http://www. crd.york.ac.uk/PROSPERO/display_record.asp?ID= CRD42016037321. Accessed 11 Nov 2016

36. Tluczek A, Henriques JB, Brown RL (2009) Support for the reliability and validity of a six-item state anxiety scale derived from the State-Trait Anxiety Inventory. J Nurs Meas 17(1):19

37. Beck AT, Epstein N, Brown G, Steer RA (1988) An inventory for measuring clinical anxiety: psychometric properties. J Consult Clin Psychol 56(6):893

38. Zigmond AS, Snaith RP (1983) The hospital anxiety and depression scale. Acta Psychiat Scand 67(6):361-370

39. Spitzer RL, Williams JB, Gibbon M, First MB (1992) The Structured Clinical Interview for DSM-III-R (SCID) I: history, rationale, and description. Arch Gen Psychiatry 49(8):624

40. National Institute for Health and Care Excellence (NICE) (2016) Early and locally advanced breast cancer overview. NICE, London. http://pathways.nice.org.uk/pathways/early-and-locally-advancedbreast-cancer/early-and-locally-advanced-breast-cancer-overview\# content=view-node:nodes-follow-up-care. Accessed 11 Nov 2016

41. Khatcheressian JL, Hurley P, Bantug E, Esserman LJ, Grunfeld E, Halberg F et al (2013) Breast cancer follow-up and management after primary treatment: American Society of Clinical Oncology clinical practice guideline update. J Clin Oncol 31(7):961-965

42. Runowicz CD, Leach CR, Henry NL, Henry KS, Mackey HT, Cowens-Alvarado RL et al (2016) American Cancer Society/ American Society of Clinical Oncology Breast Cancer Survivorship Care Guideline. CA Cancer J Clin 66(1):43-73

43. Saboonchi F, Petersson L-M, Wennman-Larsen A, Alexanderson K, Vaez M (2015) Trajectories of anxiety among women with breast cancer: a proxy for adjustment from acute to transitional survivorship. J Psychosoc Oncol 33(6):603-619

44. Fielding R, Lam W (2014) Psychosocial and physical outcomes after surgery for breast cancer: 5-to-6-year follow-up. Hong Kong Med J 20(6 Supplement 7)

45. Gaugler JE, Duval S, Anderson KA, Kane RL (2007) Predicting nursing home admission in the US: a meta-analysis. BMC Geriatr 7(1):1

46. Bours MJ, van der Linden BW, Winkels RM, van Duijnhoven FJ, Mols F, van Roekel EH et al (2016) Candidate predictors of healthrelated quality of life of colorectal cancer survivors: a systematic review. Oncologist 21(4)

47. Guyatt GH, Oxman AD, Schünemann HJ, Tugwell P, Knottnerus A (2011) GRADE guidelines: a new series of articles in the Journal of Clinical Epidemiology. J Clin Epidemiol 64(4):380-382

48. Hill J, Holcombe C, Clark L, Boothby MRK, Hincks A, Fisher J et al (2011) Predictors of onset of depression and anxiety in the year after diagnosis of breast cancer. Psychol Med 41(7):1429-1436 
49. Burgess C, Cornelius V, Love S, Graham J, Richards M, Ramirez A (2005) Depression and anxiety in women with early breast cancer: five year observational cohort study. BMJ 330(7493):702-705

50. Saboonchi F, Petersson L-M, Wennman-Larsen A, Alexanderson K, Brannstrom R, Vaez M (2014) Changes in caseness of anxiety and depression in breast cancer patients during the first year following surgery: patterns of transiency and severity of the distress response. Eur J Oncol Nurs 18(6):598-604

51. Hopwood P, Sumo G, Mills J, Haviland J, Bliss JM, Group STM (2010) The course of anxiety and depression over 5 years of followup and risk factors in women with early breast cancer: results from the UK Standardisation of Radiotherapy Trials (START). Breast 19(2):84-91

52. Dean C (1987) Psychiatric morbidity following mastectomy: preoperative predictors and types of illness. J Psychosom Res 31(3):385-392

53. Moreira H, Canavarro MC (2010) A longitudinal study about the body image and psychosocial adjustment of breast cancer patients during the course of the disease. Eur J Oncol Nurs 14(4):263-270

54. Lam WWT, Shing YT, Bonanno GA, Mancini AD, Fielding R (2012) Distress trajectories at the first year diagnosis of breast cancer in relation to 6 years survivorship. Psycho-Oncology 21(1):90-99

55. Baron RM, Kenny DA (1986) The moderator-mediator variable distinction in social psychological research: conceptual, strategic, and statistical considerations. J Pers Soc Psychol 51(6):1173
56. Hopwood P, Haviland J, Mills J, Sumo G, Bliss JM (2007) The impact of age and clinical factors on quality of life in early breast cancer: an analysis of 2208 women recruited to the UKSTART Trial (Standardisation of Breast Radiotherapy Trial). Breast 16(3):241-251

57. Bonnet F, Irving K, Terra J-L, Nony P, Berthezène F, Moulin P (2005) Anxiety and depression are associated with unhealthy lifestyle in patients at risk of cardiovascular disease. Atherosclerosis 178(2):339-344

58. Grimmett C, Bridgewater J, Steptoe A, Wardle J (2011) Lifestyle and quality of life in colorectal cancer survivors. Qual Life Res 20(8):1237-1245

59. Kanera IM, Bolman CA, Mesters I, Willems RA, Beaulen AA, Lechner L (2016) Prevalence and correlates of healthy lifestyle behaviors among early cancer survivors. BMC Cancer 16(1): 1

60. Gopalakrishna A, Longo TA, Fantony JJ, Van Noord M, Inman BA (2016) Lifestyle factors and health-related quality of life in bladder cancer survivors: a systematic review. J Cancer Surviv:1-9

61. McNamee R (2005) Regression modelling and other methods to control confounding. Occup Environ Med 62(7):500-506

62. Moons KG, Altman DG, Reitsma JB, Ioannidis JP, Macaskill P, Steyerberg EW et al (2015) Transparent Reporting of a multivariable prediction model for Individual Prognosis Or Diagnosis (TRIPOD): explanation and elaboration. Ann Intern Med 162(1): W1-W73 